\title{
Alterations of humoral, cell mediated and antibody dependent cell mediated cytotoxic responses during the course of amoebic infection in guinea pigs
}

\author{
V K VINAYAK, A SAXENA, AND A K MALIK \\ From the Division of Experimental Parasitology and Parasitic Immunology, Department of Experimental \\ Medicine, and Department of Gastroenterology, Post Graduate Institute of Medical Education and Research, \\ Chandigarh, India
}

SUMmaRY The cellular and antibody dependent cellular cytotoxic (ADCC) responses of splenic lymphocytes and peritoneal macrophages obtained from animals at variable intervals after inoculation were studied against trophozoites of axenic $E$ histolytica (NIH:200). Cytotoxic responses of effector cells from infected animals were compared with those of effector cells from vaccine stimulated and unstimulated uninfected control animals. Cellular and antibody-dependent cellular cytotoxic responses of the effector cells from animals during the establishment and acute phase of infection were significantly suppressed, compared with unstimulated uninfected and vaccinated (FI amoebic proteins stimulated) effector cells. The effector cells from animals recovered from infection showed enhanced cytotoxic responses against trophozoites of $E$ histolytica. The suppressed cytotoxic response was accompanied by impairment of cytotoxic cell activities and lack, or very low level of anti-FI antibodies in the sera of animals during the establishment phase of infection. With the rise in anti-FI antibodies in the sera of animals ADCC could be induced effectively against trophozoites of $E$ histolytica, which seem to result in clearance of amoebic infection.

\begin{abstract}
Amoebiasis is a disease caused by a protozoan parasite Entamoeba histolytica. It is world wide in distribution. According to a recent estimate,' 480 million people carried $E$ histolytica in their intestinal tract. Of these, 48 million people suffered from acute amoebic disease. Amoebiasis thus is an important human parasitic infections specially in tropical and subtropical countries. Mass examination followed by selective chemotherapy can not be considered a solution to this problem. Immunological intervention, therefore, seems logical. A WHO expert group 2 in an informal meeting on 'Strategies for controls of amoebiasis' emphasised the urgency to elucidate cellular and humoral immune responses in relation to immunoprotection against amoebiasis.
\end{abstract}

\footnotetext{
Address for correspondence: 1)r V K Vinayalk. Associate Professor, Department of Experimental Medicine. Post Graduate Institute of Medical Education and Rescarch, Chandigarh - 1601)12, India.

Received for publication 19 March 1987.
}

Efforts have been made during last two decades to develop a suitable vaccine against $E$ histolytica. Varying degree of success has been reported using crude amoebic extracts ${ }^{i t}$ and a partially purified fraction (FI) of crude amoebic extract ${ }^{t h}$ as vaccines in experimental animals. It is well known that an intense humoral immune response is mounted against amoebic antigens during hepatic infection ${ }^{7-11}$ whereas cellular immune responses have been shown to be suppressed. " "I" The importance of cell mediated immune responses is also evident from the results of experiments involving experimental immunosuppression of rats, guinea pigs and hamsters leading to exageration of amoebic lesions. ${ }^{12-14}$ Ghadirian and Meerovitch ${ }^{15}$ showed that immunity could be passively transferred with immune spleen cells and peritoneal exudate cells to normal hamsters. It is not yet clear, however, whether humoral or, cellular immune responses or a combina- 
tion of both would be required in controlling amoebic infection. Our recent investigation on antibody dependent cellular cytotoxicity (ADCC) against $E$ histolytica in vitro have indicated that both humoral as well as cellular immune responses may be important in controlling amoebic infection. ${ }^{1{ }^{14}-19}$ Antibody dependent cellular cytotoxicity, a manifestation of both cellular and humoral immune responses, has been shown to be operative against other parasites also." Although the study of ADCC has provided new insights into immune defence against parasites, direct evidence of in vivo role of this mechanism is largely lacking. In order to assess the role of cellular and antibody dependent cellular cytotoxicity in development and control of amoebic infection, we studied the alteration of these effector mechanisms during the course of experimental amoebic infection in guinea pigs.

\section{Methods}

\section{PARASITES}

An axenic isolate Entamoeba histolytica (NIH:200) was used for preparation of antigens. The trophozoites of $E$ histolytica (NIH:200) were used as target cells in the cytotoxicity assays. A polyaxenic, moderately virulent isolate of E histolytica (SB) was used for infecting animals.

\section{ANTIGENS}

Crude amoebic extract (CAE) of axenic E histolytica (NIH:200) was fractionated as described previously." ${ }^{21}$ The highest molecular weight $(>650000 \mathrm{~d})$ fraction (FI) was used for immunisation of guinea pigs.

\section{ANIMALS}

Three to four weeks old guinea pigs free from natural infection were divided into six groups four of which were infected intracaecally after laparotomy with $80000 \pm 20000$ amoebic trophozoites of isolate of SB. ${ }^{22}$

Group 1 (six animals): Uninfected and unimmunised control animals; group 2 (six animals): Immunised with FI emulsified with complete Freund's adjuvant; group 3 (six animals): Infected and killed three days postinoculation; group 4 (six animals): Infected and killed seven days postinoculation; group 5 (six animals): Infected and killed 14 days postinoculation; group 6 (six animals): Infected and killed 21 days postinoculation.

On the specific postinoculation day animals were bled by cardiac puncture and killed by ether narcosis.

IMMUNOLOGICAL INVESTIGATIONS

Anti-CAE and anti-FI-antiamoebic antibody titres were determined using $\mathrm{CAE}$ and $\mathrm{FI}$ amoebic proteins as antigens respectively at various days postinoculation by the micro indirect haemagglutination (IHA) method. ${ }^{+}$All antisera were heat inactivated at $56^{\circ} \mathrm{C}$ for 30 minutes. Leucocyte migration inhibition (LMI) responses to amoebic proteins (FI) and phytohaemagglutinin (PHA) was studied essentially as

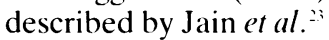

COLLECTION OF EFFECTOR CELLS

Spleen cells were layered on Ficoll-paque (Pharmacia, Sweden) and centrifuged at $2000 \mathrm{rpm}$ for 20 minutes. Mononuclear cells (MNC) were collected from the interface of gradient and the medium and washed twice with cold MEM. Cell concentration was adjusted to $10 \% / \mathrm{ml}$.

Resident macrophages were collected by injecting cold MEM into the peritoneum of guinea pigs and macrophage monolayers were prepared in specially designed chambers." The macrophages were characterised by giemsa staining, Fc receptor expression and acid phosphatase activity and found to be $98 \%$ pure.

\section{CYTOTOXICITY ASSAY}

The cytotoxicity assay was carried out by mixing effector cells - that is, splenic MNC or peritoneal resident macrophages and trophozoites of $E$ histolytica as described earlier ${ }^{11}{ }^{1 "}$ using trypan blue dye exclusion method. The cytotoxic potentialities of both the types of effector cells - that is, splenic MNC and peritoneal macrophages obtained from unimmunised uninfected, infected and FI stimulated animals were studied with normal guinea pig serum (NGPS), heat inactivated hyperimmune anti-FI antiserum and serum samples obtained from guinea pigs at various days post inoculation. Each assay was done in duplicate and the data were analysed by Student's $t$ test.

\section{PARASITOLOGICAL EXAMINATION}

At the time of death, the caecum of each animal was examined for the presence of macroscopic lesions and the presence of trophozoites in the caecal contents. Caecal tissues with lesions were fixed in $10 \%$ formal saline, processed and stained both by $\mathrm{H} \& \mathrm{E}$ and PAS methods.

\section{Results}

COURSE OF AMOEBIC INFECTION

The animals infected with moderately virulent polyxenic isolate of $E$ histolytica were killed at days 3, 7,14 , and 21 postinoculation (PI). The parasitological examination revealed that the trophozoites were present in the ceacal content at day $3 \mathrm{PI}$ in all the 
animals. Histopathological examination of caecal tissues confirmed the presence of parasite which were found just invading the villi (Fig. 1a). Minimum to no invasion was observed at this stage. Animals killed at day 7 PI, however, not only had trophozoites in the caecal contents, the parasite had also invaded the gut epithelium (Fig. 1b) and the caecum showed typical amoebic lesions (Fig. 1c). At day 14 PI although the trophozoites were still present in the caecal content, minimum (35\% of animals) to no invasion was observed. Trophozoites were absent from the caecal content as well as caecal tissues by day 21 PI (Fig. 1d) indicating the clearance of infection.

HUMORAI. IMMUNE RESPONSE

Figure 2 shows anti-CAE and anti-FI antibody titres by IHA during the course of amoebic infection. The antiamoebic antibodies were not detectable by IHA at day 3 PI. The anti-CAE and anti-FI-geometric mean titre (GMT) at day 7 PI were 45 and 14 respectively which increased to 64 and 32 respectively by day $21 \mathrm{PI}$. The rise in anti-FI titres was accompanied by invasion and subsequent clearing of the parasite by the animals.

\section{CELLULAR IMMUNE RESPONSE}

Figure 2 also shows leucocyte migration inhibition (LMI) response to specific amoebic antigen (FI) and PHA during the course of amoebic infection. Response to FI amoebic proteins in terms of LMI indices increased gradually from $19 \cdot 2 \pm 3 \cdot 5 \%$ at day 3 PI to $36 \cdot 6 \pm 3 \cdot 4 \%$ at day $21 \mathrm{PI}$ whereas the response to PHA decreased initially from $35 \cdot 0 \pm 3 \cdot 5 \%$ at day 0 $21 \cdot 0 \pm 3.2 \%$ at day $7 \mathrm{PI}$ and then increased to $30 \cdot 5 \pm 6 \cdot 5 \%$ at day $21 \mathrm{PI}$.

\section{ALTERATION OF CELLULAR AND ANTIBODY} DEPENDENT CELLULAR CYTOTOXICITY

The Table shows that the induction of anti-FI antibody dependent $\mathrm{MNC}$ mediated cytotoxic response
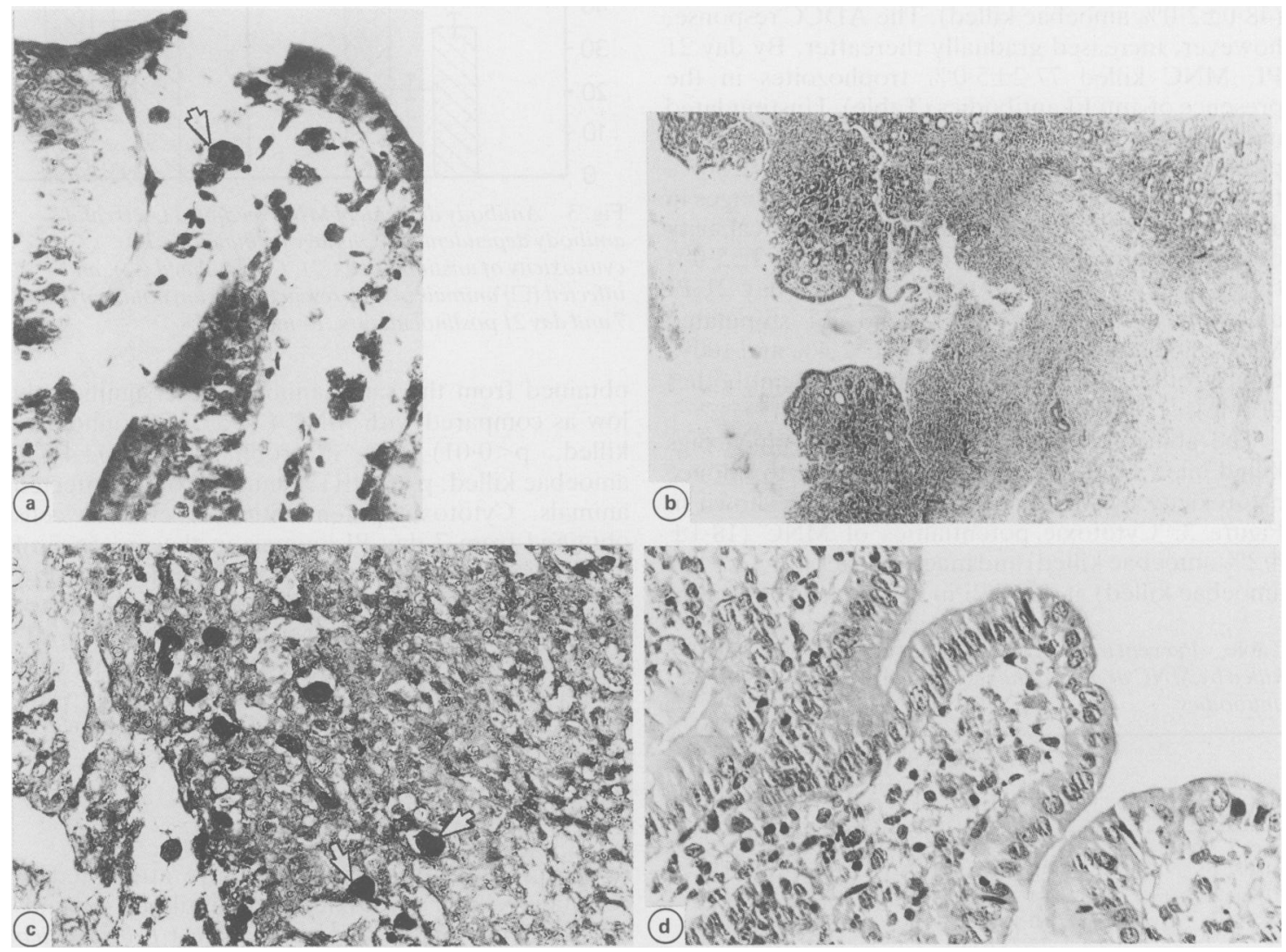

Fig. 1 Course of intestinal amoebic infection (a) amoebic trophozoites (arrow) can be seen entering the mucosa at day 3 PI, (b) a typical flask shaped ulcer at day $7 P I$, (c) a deep penetrating ulcer showing large number of trophozoites (arrows), (d) the infection is cleared at day 21 PI. (PAS stained.) 


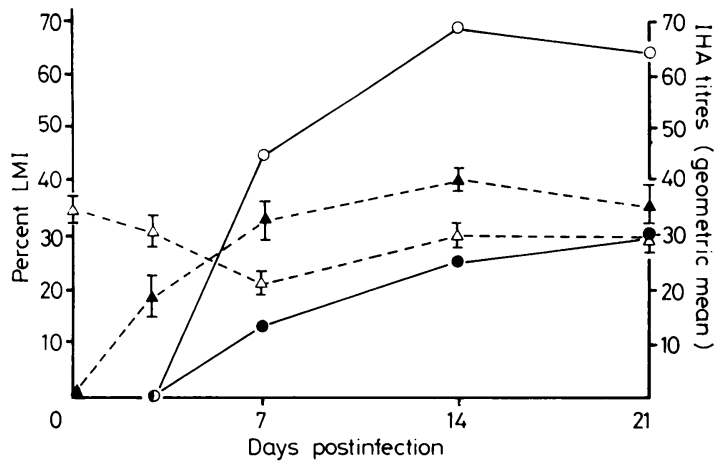

Fig. 2 Levels of antiamoebic antibodies and leucocyte migration inhibition during the course of amoebic infection. Geometric mean IHA titres, anti-CAE (O-O), anti-FI (-). Leucocyte migration inhibition using PHA $(\triangle-\triangle)$ and Fl amoebic proteins $(\mathbf{\Delta}-\mathbf{\Delta})$ as antigens. $I=$ mean $\pm S D$.

at day 7 PI (34.7 $\pm 4 \cdot 3 \%$ amoebae killed) was significantly $(\mathrm{p}<0 \cdot(0) 1)$ low as compared with that at day 0 $(48 \cdot 0 \pm 2 \cdot(\%)$ amoebae killed). The ADCC response, however, increased gradually thereafter. By day 21 PI, MNC killed $77 \cdot 2 \pm 5.0 \%$ trophozoites in the presence of anti FI antibodies (Table). Unstimulated macrophages killed $98.0 \pm 2.5 \%$ trophozoites in the presence of anti-FI antibodies while only $40 \cdot 7 \pm 3 \cdot 6 \%$ trophozoites were killed by day 7 PI macrophages in association with anti-FI antibodies. Killing capacity of macrophages also increased thereafter; $77 \cdot 2 \pm 5 \cdot 0 \%$ trophozoites were found to be killed by day $21 \mathrm{PI}$ macrophages. On the other hand, FI stimulated MNC and macrophages killed $78 \cdot 0 \pm 2 \cdot 4 \%$ and $100 \%$ trophozoites in the presence of anti-FI antibodies (Table).

The ability of serum obtained from guinea pigs killed at various days postinoculation to induce cytotoxicity by $\mathrm{MNC}$ and macrophages is shown in Figure 3. Cytotoxic potentialties of MNC (18.1 $4 \cdot 2 \%$ amoebae killed) and macrophages $(23 \cdot 3 \pm 2 \cdot 9 \%$ amoebae killed) at day 7 PI in the presence of serum

Table Percent trophozoites of E histolytica (NIH:200) killed by MNC or macrophages in the presence of anti-FI antibodies

\begin{tabular}{llc}
\hline & \multicolumn{2}{l}{$\%$ Trophozoites killed by } \\
\cline { 2 - 3 } Days PI & $M N C$ & Macrophages \\
\hline 0 & $48 \cdot() \pm 2 \cdot 0$ & $98 \cdot() \pm 2 \cdot 5$ \\
3 & $36 \cdot 98 \pm 2 \cdot 34^{*}$ & $75 \cdot 47 \pm 4 \cdot 56$ \\
7 & $34 \cdot 73 \pm 4 \cdot 33$ & $4\left(\cdot \cdot 76 \pm 3 \cdot 68^{*}\right.$ \\
14 & $6(11 \pm 3 \cdot(16$ & $6(0) \cdot 33 \pm 4 \cdot 12$ \\
21 & $77 \cdot 23 \pm 5 \cdot(01$ & $77 \cdot 20 \pm 5 \cdot(01$ \\
FI stimulated & $78 \cdot() \pm 2 \cdot 5$ & $1(0) \cdot 0$ \\
\hline
\end{tabular}

${ }^{*} p<0 .(0) 1$ as compared with day 0.
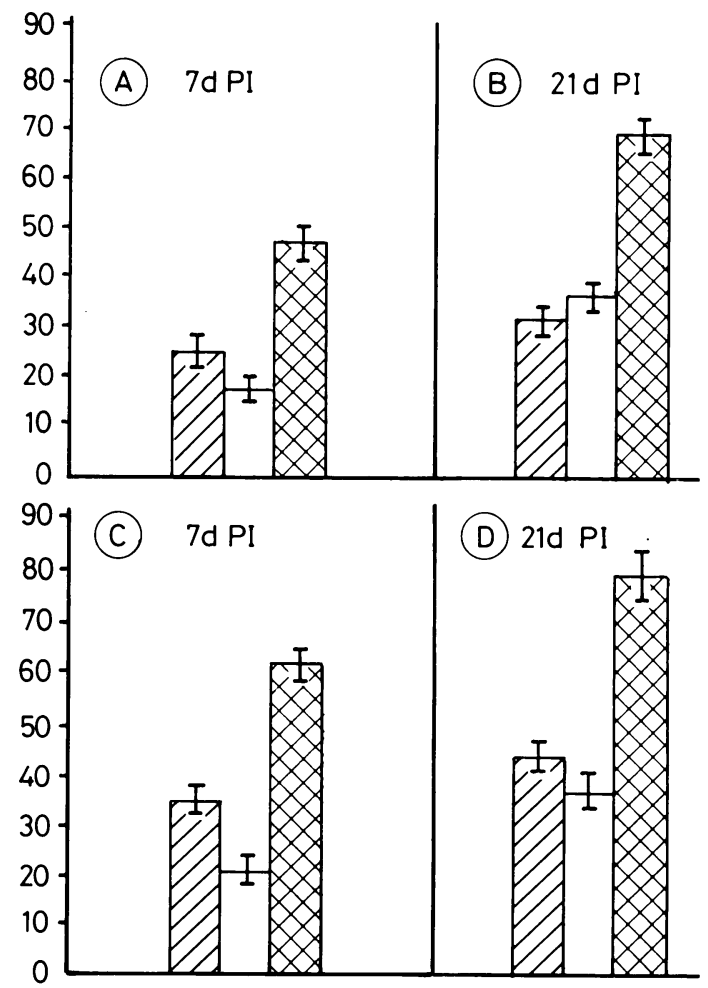

Fig. 3 Antibody dependent MNC mediated $(A, B)$ and antibody dependent macrophage mediated $(C, D)$ cytotoxicity of unstimulated (..), FI stimulated (\%), and infected ( $\square$ ) animals in the presence of serum obtained at day 7 and day $2 I$ postinoculation. $I=$ mean $\pm S I$ ).

obtained from the same animals were significantly low as compared with MNC $(24 \cdot 2 \pm 3 \cdot 3 \%$ amoebac killed, $p<0 \cdot(01)$ and macrophages $(45 \cdot() \pm 4.5 \%$ amoebae killed, $\mathrm{p}<0 \cdot(0) 1)$ obtained from uninfected animals. Cytotoxic potentialities of effector cells obtained from 7 day PI animals in the presence of homologous serum was also significantly low $(p<0 \cdot 001)$ as compared with FI stimulated effector cells under similar conditions (Fig. 3a, c). By day 21 , however, PI cytotoxic capacity of effector cells increased and was found to be comparable with normal effector cells (Fig. 3b, d) in the presence of day 21 PI serum.

\section{Discussion}

In the present investigation we have attempted to correlate cellular cytotoxicity and antibody dependent cellular cytotoxic responses against $E$ histolytica in vitro with the diseased state and immunoprotected state of guinea pigs. It is well known that FI immunised animals are protected to a very high degree 
against experimental challenge with highly virulent isolates of $E$ histolytica. ${ }^{+\infty}$ Thus, FI stimulated animals represent protected state. The use of a moderate virulent challenge strain of $E$. histolytica enabled us to show three phases of infection - that is, establishment of infection (0) to 3 days PI), the acute phase (three to seven days PI) and clearing of infection by the animals (14-21 days PI). Histopathology showed that amoebae had broken through the mucosa and submucosa where a great number of amoebic trophozoites could be seen. The submucosa showed marked oedema and a collection of polymorphs, mononuclear histiocytes and lymphocytes. This histopathological picture is similar to that found in human intestinal lesions except that cellular infiltration is relatively less marked in the latter.

The immune responses were monitered in all phases. It was observed that the establishment of amocbic infection was accompanied by suppression of LMI response to PHA. At day $7 \mathrm{PI}$ when the infection was in the acute phase, response to PHA was found to be lowest. On the other hand, LMI response to specific Sephadex G-200) chromatographed FI of crude amoebic extract increased gradually with the establishment of infection and so did the antiamoebic antibody titres. The increasing sensitisation of host cells and the appearance of antiamoebic anti-FI antibodies were accompanied by invasion of host tissues by the parasite. This increasing trend continued until day $14 \mathrm{PI}$ and was stabilised thereafter. The response to PHA also showed an increasing trend after day $7 \mathrm{PI}$ and reached normal levels by day $21 \mathrm{PI}$. These observations confirm those of others who showed a good response to antigen while suppressed response to PHA in acute amoebic infection." "'" The suppression of the ability of effector cells to kill the parasite in vitro during the establishment phase parallels the lowered response to PHA. We have shown here that with clearing of amoebic infection, the cell mediated immune responses are gradually restored to normal. Cellular cytotoxicity and ADCC of both MNC and macrophages, a functional manifestation of CMI and HMI showed significant decline during the first seven days PI followed by increased ADCC activities which was accompanied by gradual clearing of amoebic infection. FI stimulated effector cells on the other hand showed significantly higher cytotoxic responses against E histolytica. Therefore the ability of effector cells to kill trophozoites of E histolytica correlates with the diseased state because these responses were suppressed in the acute phase of infection and also with the immuneprotected state of guinea pigs. Our results correlate with others who showed that effector cells obtained from vaccinated hamsters ${ }^{2+}$ and gerbils ${ }^{-5}$ could kill trophozoites of Ehistolytica in vitro. As ADCC involved both effector cells and antiamoebic antibodies, we sought to determine whether impairment of ADCC was caused by suppression of cells or was because of the inability of host antibodies to induce cytotoxicity or both. It was observed that both unstimulated lymphocytes and macrophages showed increasing capacity to kill trophozoites with increasing anti-FI antiamoebic antibody titres obtained at different days postinoculation indicating that such antibodies from infected animals were capable of inducing cytotoxicity. The effector cells obtained at different days PI on the other hand, failed to kill a significant number of parasites in vitro in the presence of homologous and hyperimmune anti-FI antibodies until day 7 PI. Thereafter, both the effector cells - that is, splenic lymphocytes and peritoneal macrophages of infected animals, could kill sufficient number of trophozoites which was accompanied subsequently by clearing of infection by such animals. The cytotoxic potentialities of effector cells obtained from infection stimulated animals, however, were not comparable with those of FI stimulated animals probably because the levels of anti-FI antibodies achieved in immunised animals is much higher than that obtained during intestinal infection. It has been shown recently ${ }^{20.27}$ that in the patients after amoebic liver abscess, specific cell mediated immune mechanism develop that are effective in vitro against the parasite. It is also well known that patients successfully cured of amoebic liver abscess rarely develop a second infection $^{2 \times 1}$ indicating the development of immunity against this form of the disease. The question whether this immunity is a result of enhanced cellular and antibody dependent cellular cytotoxic responses is open to debate. The observations that experimental immunosuppression with ALS and corticosteroids exaggerated amoebic lesions in experimental animals favour this correlation. The exaggeration of amoebic lesion has also been reported in patients on costecosteriod therapy. ${ }^{29}$.i) Lack of cellular responses in patients suffering from amoebiasis, however, are against it.

We feel that establishment of amoebic infection is accompanied by suppression of cellular immune response as reflected by suppressed cellular cytotoxicity both of $\mathrm{T}$ lymphocytes and macrophages reaching their lowest level during the acute phase. The rising antiamoebic antibodies particularly antiFI antibodies and restoration of cytotoxic responses against trophozoites direct the infection to recede and eventually clear from the animals. During the establishment phase both absence or low level of anti-FI antibodies and suppressed cytotoxic responses make it possible for the trophozoites to invade and establish in the host. 
The Indian Council of Medical Research is gratefully acknowledged for financial assistance.

\section{References}

1 Walsh JA. Epidemiology and magnitude of the problem of amoebiasis. A magnitude of the problem (morbidity and mortality on a global scale). Workshop on the World Problem of Amoebiasis. Current Status Research Needs and Opportunities for advancement. Prospect Hill 16-18 June 1982, 14.

2 World Health Organization. Informal meeting on strategies for control of amoebiasis. Geneva: WHO, 1984.

3 Krupp IM. Protective immunity to amocbic infection demonstrated in guinea pigs. Am J Trop Med Hyg 1974; 23: $355-60$

4 Vinayak VK, Sawhney S, Jain P, Chugh S, Chakravarti RN. Protective effect of crude and chromatographic fraction of axenic. Entamoeba histolytica in guinea piga. Trans R Soc Trop Med Hyg 1980; 74: 483-7.

5 Ghadirian E, Meerovitch E, Hartman DP. Protection against amoebic liver abscess in hamsters by means of immunization with amoebic antigen and some of its fractions. Am J Trop Med Hyg 1980; 29: 779-84.

6 Sepulveda B, Diamond LS, eds. Immunology of amoebiasis. Mexico: Inst Mexicano del Seguro Social Mexico, 1975: 186-702.

7 Kessel JF, Lewis WP, Pasquel CM, Turner JA. Indirect haemagglutination and complement fixation tests in amoebiasis. Am J Trop Med Hyg 1965; 14: 540-50.

8 Gold D, Norman LG, Maddison SE, Kagan IG. Immunologic studies on hamsters infected with Entamoeba histolytica. J Parasitol 1978; 64: 866-73.

9 Vinayak VK. The specificity of indirect hemagglutination test in the immunodiagnosis of amoebiasis. Ind $J$ Prev Soc Med 1975; 6: 271-6.

10 Vinayak VK, Jain P, Gupta B, Kaushik SP, Sawhney S. Cellular and humoral immune responses in amoebic patients. Trop Geogr Med 198(); 32: 298-3()2.

11 Simjee AE, Gathiram V, Coovadia HM, et al. Cell mediated immunity in hepatic amoebiasis. Trans Roy Soc Trop Med Hyg 1985; 79: 165-8.

12 Vinayak VK, Chitkara NL, Chhuttani PN. Effect of corticosteroid and irradiation on caecal amoebic infection in rats. Trans Roy Soc Trop Med Hyg 1979; 73: 266-8.

13 Vinayak VK, Sawhney S, Jain P, Chakravarti RN. Immunosuppression and experimental amoebiasis in guinea pigs. Trans Roy Soc Trop Med Hyg 1982; 76: 309-16.

14 Tanimoto-weki M, Calderon P, De La Hoz R, Aguirre Galca J. Inoculation of Entamoeba histolytica trophozoites in hamsters on immunosuppressive therapy. Arch Invest Med 1974; 5: 441-6.

15 Ghadirian E. Meerovitch E. Passive transfer of immunity against hepatic amocbiasis in the hamsters by cells. Parasit Immunol 1983; 5: 369-76.

16 Vinayak VK. Chugh S. Saxena A. Sharma SP. Antibody dependent lymphocyte mediated cytotoxicity in amoebiasis. Ind J Med Res 1984: 80: 421-7.

17 Chugh S. Saxena A. Vinayak VK. Interactions between trophozoites of Entamoeba histolytica and cells of the immune system. Aust J Exp Biol Med Sci 1985; 63: 1-8.

18 Saxena A, Chugh S, Vinayak VK. Elucidation of cellular population and nature of antiamoebic antibodics in cytotoxicity to Entamoeba histolvtica (NIH:200). J Parasitol 1986; 72: 434-8.

19 Saxena A, Chugh S, Vinayak VK. Antibody dependent macrophage mediated cytotoxicity against Entamocha histolytica. J Med Microbiol 1986; 22: 17-21.

20 Capron A. Dessaint JP. Haque A, Capron M. Antibody dependent cell mediated cytotoxicity against parasites. Prog Allergy 1982; 31: 234-67.

21 Sawhney S, Chakravarti RN, Jain P, Vinayak VK. Immunogenicity of axenic Entamoeba histolytica antigens and its fractions. Trans $R$ Soc Trop Med Hyg 1980; 74: 26-29.

22 Vinayak VK, Naik SR, Sawhney S, Sehmi N, Chhuttani PN. Pathogenicity of Entamoeba histolytica virulence of strains of amoebae from symptomatic and asymptomatic cases of amoebiasis. Ind J Med Res 1977; 66: 935-41.

23 Jain P, Sawhney S, Vinayak VK. Experimental amoebic infection in guinea pigs immunized with low grade amoebic infection. Trans $R$ Soc Trop Med Hyg 1980; 74: 347-50.

24 Ghadirian E, Meerovitch E. In vitro amocbicidal activity of immune cells. Inf Immun 1982; 36: 243-46.

25 Chadee K, Meerovitch E, Moreau F. In vitro and in vivo interaction between trophozoites of Entamoeba histolytica and Gerbil lymphoid cells. Inf Immun 1985; 49: 228-32.

26 Salata RA, Pearson RD, Ravdin JI. Interaction of human leukocyte and Entamoeba histolytica: killing of virulent amoebae by activated macrophages. J Clin Invest 1985; 76: 491-9.

27 Salata RA, Martinez-Palomo A, Murray HW, et al. Patients treated for amoebic liver abscess develop cell mediated immune responses effective in vitro against Entamoeba histolytica. J Immunol 1986; 136: 2633-9.

28 Krupp IM, Jung RC. Immunity of amoebic infection. In: Cohen S, Sadun EH, eds. Immunology of parasitic infections. Oxford: Blackwell Scientific Publications, 1976: 163-6.

29 Villarejos SVM. Corticosteroid and experimental amoebiasis in rats. J Parasitol 1962; 48: 194.

30 El-Hannawy M, Abd-Rabbo H. Hazards of cortisone therapy in hepatic amoebiasis. J Trop Med Hyg 1978;81: 71-3. 